\title{
Impact of next future Direct Detection experiments on Dark Por- tals and beyond
}

\author{
Giorgio Arcadi ${ }^{1, a}$ \\ ${ }^{1}$ Max Planck Institüt für Kernphysik, Saupfercheckweg 1, 69117 Heidelberg, Germany
}

\begin{abstract}
WIMPs are attractive DM candidates. The simplest model realizations, dubbed Dark Portals are in strong tensions with detection constraints. Two component DM setups can relax these tensions.
\end{abstract}

\section{Introduction}

Weakly interacting massive particles (WIMPs) are among the most popular DM candidates. Their production mechanism predicts the existence, in thermal equilibrium, of the DM in the primordial thermal bath and a subsequent decoupling (freeze-out). The value of the DM abundance is determined by a single particle physics input, consisting in the thermally averaged pair annihilation cross-section; more specifically $\Omega_{\mathrm{DM}} h^{2} \propto 1 /\langle\sigma v\rangle$ so that $\Omega_{\mathrm{DM}} h^{2} \simeq 0.12 \rightarrow\langle\sigma v\rangle \sim 3 \times 10^{-26} \mathrm{~cm}^{3} \mathrm{~s}^{-1}$ [1]. This value of the pair annihilation cross section implies sizeable enough interactions between the DM and the SM states to be in the reach of present experimental facilities. A simple framework for unveiling the potential correlations between the DM relic density and experimental searches is represented by the 'Dark Portals'; simple extensions of the SM in which the DM annihilations into SM states (mostly SM fermions) are induced by a s-channel mediator. In these kind of setups the DM annihilation cross-section and experimentally testable observables, like the scattering cross-section on nucleons, are related by simple crossing-symmetries. Because of these strong correlations, however, Dark Portals are in increasing tension with constraints from Direct Detection and many realizations will be substantially ruled-out in case of absence of signals from next generations of 1TON detectors, like XENON1T. A possible way-out for preserving the WIMP paradigm, compatibly with experimental constraints, is represented by considering two-component Dark portals. We will briefly illustrate a specific example in the second part of the text.

\section{Single Component Dark Portals}

Dark Portals are simple extension of the Standard model with the DM candidate and, possibly, a mediator of its interactions with the SM (fermions). Being these models built on purely phenomenological basis one can consider all the possible spin combinations between the DM and the mediator which are allowed by Lorentz invariance. For definiteness we will just focus on the cases of a scalar DM candidate $\chi$ or a vector state $V^{\mu}$ interacting with a scalar mediator. This kind of interaction does not

\footnotetext{
ae-mail: giorgio.arcadi@mpi-hd.mpg.de
} 
require, in general, the presence of extra states, with respect to the SM, since it is realized, at the renormalizable level and in a Lorentz and gauge invariant way, by the four-field interactions $\lambda_{\chi}|\chi|^{2} H^{\dagger} H$ or $\lambda_{V} V^{\mu} V_{\mu} H^{\dagger} H$, with $H$ being the SM Higgs doublet and $\lambda_{\chi, V}$ adimensional couplings, which lead, after EW symmetry breaking, to trilinear interactions between the SM Higgs and a DM pair. These kind of interactions can be schematically written as (for simplicity we report just the interactions with SM fermions):

$$
\mathcal{L}_{\chi}=\left(\mu_{\chi}|\chi|^{2}+\lambda_{f} \bar{f} f\right) h, \quad \mathcal{L}_{V}=\left(\mu_{V} V^{\mu} V_{\mu}+\lambda_{f} \bar{f} f\right) h
$$

where $\mu_{\chi, V}=\lambda_{\chi, V} v_{h}$ and $\lambda_{f}=m_{f} / v_{h}$, with $v_{h}$ being the Higgs vev. The scalar or vectorial DM annihilates into SM fermions (as well as gauge bosons and higgs pairs) through s-channel exchange of $h$. The corresponding cross sections can be analytically approximated as ${ }^{1}$ :

$$
\langle\sigma v\rangle_{\chi} \approx \sum_{f} \frac{n_{c}^{f}\left|\mu_{\chi}\right|^{2}}{4 \pi\left(m_{h}^{2}-4 m_{\chi}^{2}\right)^{2}}, \quad\langle\sigma v\rangle_{V} \approx \sum_{f} \frac{n_{c}^{f}\left|\mu_{V}\right|^{2}}{4 \pi\left(m_{h}^{2}-4 m_{V}^{2}\right)^{2}}
$$

At the same time, t-channel exchange of $h$ induces Spin Independent (SI) scattering cross-sections on nucleons of the form:

$$
\sigma_{\chi}^{\mathrm{SI}}=\frac{\left|\mu_{\chi}\right|^{2} \mu_{N_{\chi}}^{2}}{4 \pi m_{h}^{4} m_{\chi}^{2}} \frac{\left|Z f_{p}+(A-Z) f_{n}\right|^{2}}{A^{2}}, \quad \sigma_{\chi}^{\mathrm{SI}}=\frac{\left|\mu_{V}\right|^{2} \mu_{N V}^{2}}{4 \pi m_{h}^{4} m_{V}^{2}} \frac{\left|Z f_{p}+(A-Z) f_{n}\right|^{2}}{A^{2}}
$$

where $\mu_{(\chi, V) N}$ is the DM-nucleon reduced mass and $f_{p, n}$ are the couplings of the DM with protons and neutrons. A straightforward comparison between eq. (2) and (3) evidences similar parametric dependences for the two classes of cross-section. We can then compare, the constraints from the correct DM relic density and from DM DD in the bidimensional plane $\left(m_{\chi, V}, \mu_{\chi, V}\right)$. An example of this kind of comparison is shown, in fig. (1), in the case of Scalar and Vector Higgs portal (as already mentioned above we can trade, in these kind of scenarios, the dimensional parameters $\mu_{\chi, V}$ with the dimensionless quantities $\lambda_{\chi, V}$.). By imposing the constraint $\Omega_{\chi, V} h^{2}=0.12 \pm 10 \%$, it is possible to identify curves (represented in black in the two panels of fig. (1)) in the plane $\left(m_{\chi, V}, \mu_{\chi, V}\right)$. These isocontours have been overlapped to the exclusion regions obtained by imposing that the DM scattering cross-sections (3) lie below current LUX sensitivity [3] (blue regions), and a 2 year projected sensitivity of XENON1T [4] (cyan regions), and that the decay rate of the Higgs into a DM pair, when kinematically open, corresponds to an invisible branching fraction below present limits [5].

As evident, current direct detection constraints already rule-out thermal DM ad exception of the 'pole' regions, i.e. $m_{\chi, V} \simeq m_{h} / 2$ (low values of the DM mass, even outside the reach of DD experiments, are excluded since induce a too high invisible width of the Higgs). The WIMP hyphothesis would be completely ruled-out by absence of signals in XENON1T.

\section{Two component Dark Portals}

In order to keep relying on the WIMP paradigm, compatibly with DD constraints, it is necessary to decorrelate (at least partially) the DM annihilation cross-section and the scattering cross-section on nucleons. This makes necessary to go beyond the too simplistic structure of Dark Portals. An interesting possibility would be to consider a two-component DM scenario. In such a case the interplay between DM relic density and DD is more complicated. Indeed, a DD signal would arise from a sum of two, possibly different, contributions, weighted by the relative abundances of the two components. The latters are not necessarily dominated by pair annihilations into SM states, which can be directly

\footnotetext{
${ }^{1}$ These expressions are not valid close to the pole regions [2], i.e $m_{\chi, V}=m_{h} / 2$.
} 

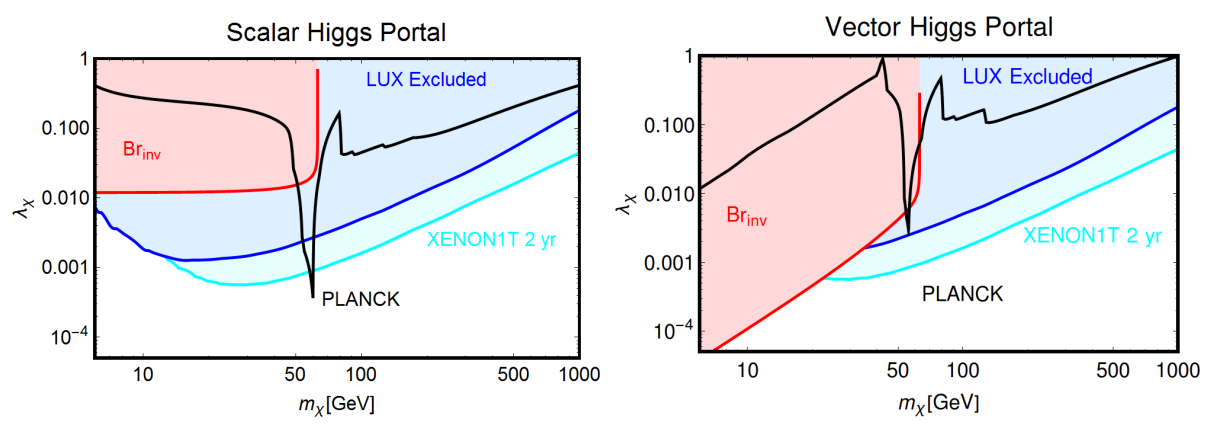

Figure 1. Combined constraints for scalar (left panel) and vector (right panel) Higgs portals. The black lines represent the isocontours of the correct DM relic density. The blue regions are excluded by current constraints by LUX [3] while the cyan region will be excluded in case of absence of signals at XENON1T after two years of excluded. In the red regions the invisible branching fraction, originated by decays into DM pairs, exceeds the experimental bounds [5].

related to the scattering rates, but processes like pair annihilations of one DM component to the other, can play a relevant role.

An interesting realization of a two-component DM scenario has been proposed in [6]. Here the SM is extended by a dark sector characterized by a new $S U(3)$ gauge symmetry, spontaneosly broken, by the vevs of two fields, $\phi_{1}$ and $\phi_{2}$, belonging to its fundamental representation, into a discrete global $\mathcal{Z}_{2} \times \mathcal{Z}_{2}^{\prime}$ symmetry. The only states, apart the higgs bosons, present in the new sector are the 8 (massive) SU(3) gauge bosons. The lightest new states odd under the discrete symmetry are automatically DM candidates. The contact between the dark and the visible (i.e. the SM) sector is established by portal like interactions induced by operators of the type $\lambda_{H 11}|H|^{2}\left|\phi_{1}\right|^{2}+\lambda_{H 22}|H|^{2}\left|\phi_{2}\right|^{2}$. The fields of the dark sector are described by the following Lagrangian:

$$
\mathcal{L}=\left(D_{\mu} \phi_{1}\right)^{\dagger}\left(D^{\mu} \phi_{1}\right)^{\dagger}+\left(D_{\mu} \phi_{2}\right)^{\dagger}\left(D^{\mu} \phi_{2}\right)^{\dagger}-\frac{1}{4} G_{\mu \nu}^{a} G^{a \mu \nu}, \quad G_{\mu \nu}^{a}=\partial_{\mu} A_{v}^{a}-\partial_{\nu} A_{\mu}^{a}-\tilde{g} f^{a b c} A_{\mu}^{b} A_{v}^{c}
$$

where the Higgs triplets $\phi_{1}$ and $\phi_{2}$ are expressed, in the unitary gauge, as:

$$
\phi_{1}=\frac{1}{\sqrt{2}}\left(\begin{array}{c}
0 \\
0 \\
v_{1}+\varphi_{1}
\end{array}\right), \quad \phi_{2}=\frac{1}{\sqrt{2}}\left(\begin{array}{c}
0 \\
v_{2}+\varphi_{2} \\
v_{3}+\varphi_{3}+i\left(v_{4}+\chi\right)
\end{array}\right)
$$

Under the assumption of CP-conservation the mixing with the Higgs doublet $H=\left(0 \frac{v+h}{\sqrt{2}}\right)^{T}$ gives rise to $4 \mathrm{CP}$-even scalars $h_{i=1,4}$ and on CP-odd $\chi$. The latter is odd also under the residual symmetry $\mathcal{Z}_{2} \times \mathcal{Z}_{2}^{\prime}$ and can then represent a DM candidate.

Despite the general model is rather complicated (see [6] for more details), its most relevant features can be caught by the following simplified limit. Assuming a direct coupling of only $\phi_{2}$ with the Higgs, i.e. $\lambda_{H 11}=0$ and setting $v_{3} \sim 0, v_{2} \ll v_{1}$, the relevant particle spectrum of the dark sector reduces to a two component DM, consisting into CP-odd dark Higgs $\chi$ and the mass degenerate vector pair $A_{1,2}^{\mu}$, interacting with the SM through the two lightest CP-even mass eigenstates $h_{1,2}\left(h_{1}\right.$ is identified with the SM Higgs). The DM interaction Lagrangian is thus simply written as:

$$
\mathcal{L}=\frac{\tilde{g} m_{A}}{2}\left(-h_{1} \sin \theta+h_{2} \cos \theta\right) \sum_{a=1,2} A_{\mu}^{a} A^{a \mu}+\lambda_{2} v_{2}\left(-\sin \theta h_{1}+\cos \theta h_{2}\right)+\lambda_{H 22} v_{h}\left(\cos \theta h_{1}+\sin \theta h_{2}\right) \chi^{2}
$$


with $\tilde{g}$ and $\theta$ being the new gauge coupling and the mixing angle between SM and dark Higgs, and substantially resembles a two-component dark portal Lagrangian. The coupling of the scalar DM component $\chi$ depends on the $\lambda_{H 22}$ and on the quartic self-coupling of $\phi_{2}, \lambda_{2}$. These can be rexpressed as:

$$
\lambda_{2}=\tilde{g}^{2} \frac{\cos ^{2} \theta m_{h_{2}}^{2}+\sin ^{2} \theta m_{h_{1}}^{2}}{4 m_{A}^{2}}, \quad \lambda_{H 22}=\tilde{g} \frac{\left(m_{h_{1}}^{2}-m_{h_{2}}^{2}\right) \sin \theta \cos \theta}{2 v_{h} m_{A}}
$$

Despite the similarity with the Lagrangians (1) the DM phenomenology is sensitively different. We have indeed that the annihilation of the vectorial component is enhanced by the presence of the 'dark' channel $A A \rightarrow \chi \chi$ which is dominant, at least in some regions of the parameter space, because does on depend, contrary to the ones into SM states, on $\sin \theta$, which is constrained to be small because of the constraints on not standard decay branching fractions of the $125 \mathrm{GeV}$ Higgs. For what regards the DM direct detection the scalar component features, instead, a peculiar behaviour. In the non relativistic limit, its effective coupling with the quarks is given by:

$$
g_{\chi \chi q q} \propto\left[\frac{1}{m_{h_{1}}^{2}}\left(\cos ^{2} \theta \lambda_{H 22} v_{h}-\sin \theta \cos \theta \lambda_{2} v_{2}\right)-\frac{1}{m_{h_{2}}^{2}}\left(\sin ^{2} \theta \lambda_{H 22} v_{h}+\sin \theta \cos \theta \lambda_{2} v_{2}\right)\right]
$$

It can be straightforwardly verified, by substituting eq. (7), that this coupling is null.

An interesting setup thus emerges: only one dark matter component is actually capable of scattering with nucleons but his abundance is reduced by a new annihilation channel, so that the total event rate at a DD experiment would be reduced. Moreover the annihilation and scattering cross-section are not fully correlated since the latter depends on $\sin \theta$ while $A A \rightarrow \chi \chi$ process not. The other DM component, with an unsuppressed abundance, is instead not capable of scattering off-nuclei.
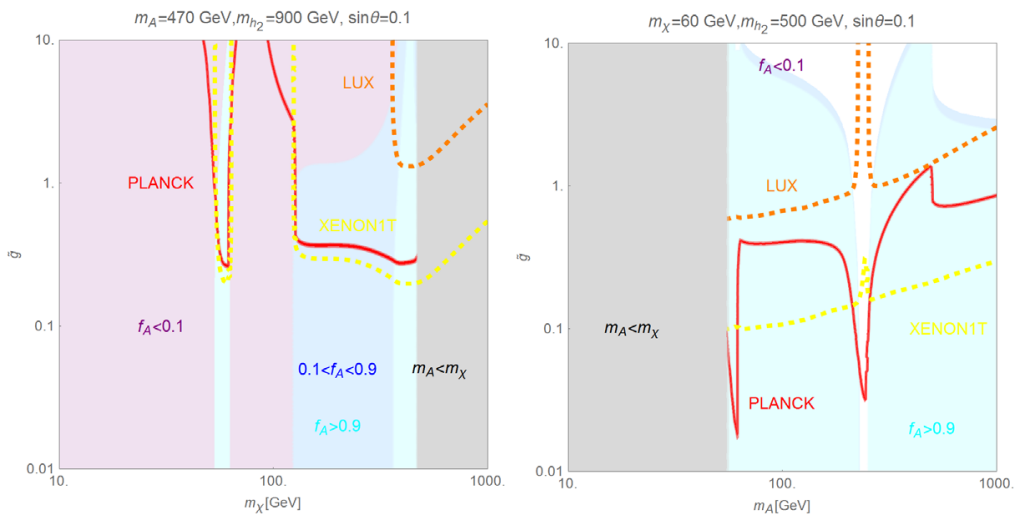

Figure 2. Combined constraints for two two-component DM benchmarks. The red contours represent the experimentally favoured value of the (total) DM relic density. The orange and yellow lines represent, respectively, the current limit by LUX [3] and a 2 yr exposure projected sensitivity by XENON1T [4]. The colored regions represent three ranges, $<0.1,0.1-0.9$ and $>0.9$, of the density fraction $f_{A}$ of the vectorial DM component.

The phenomenological impact of these features is shown on fig. (2). Here for two benchmarks we have compared, similarly to what done in fig. 1, the constraints from DM relic density and DD as function of the DM coupling (it is $\tilde{g}$ for both components) and DM mass (one mass is varied and the other kept fixed, alternatively, in the two panels.). As evident bounds from LUX do not affect the region at the correct DM relic density, even when this is dominated by the vector component (see 
right panel of fig. 2). On the contrary this scenario will be probed, at least partially by XENON1T, although an exposure time of two years will be needed.

\section{Conclusions}

WIMPs are an attractive class of DM candidates. The simplest model realizations, dubbed dark portals, are in increasing tension with limits from Direct Detection. We have shown in an explicit example that this kind of bounds can be easily relaxed in multi-component DM constructions.

Acknowledgements: The author warmly thanks the organizers of the RICAP conference for the chance of giving this contribution.

\section{References}

[1] P.A.R. Ade et al. (Planck), Astron. Astrophys. 594, A13 (2016), 1502.01589

[2] K. Griest, D. Seckel, Phys. Rev. D43, 3191 (1991)

[3] D.S. Akerib et al. (LUX), Phys. Rev. Lett. 116, 161301 (2016), 1512.03506

[4] E. Aprile et al. (XENON), JCAP 1604, 027 (2016), 1512.07501

[5] G. Aad et al. (ATLAS, CMS), JHEP 08, 045 (2016), 1606.02266

[6] C. Gross, O. Lebedev, Y. Mambrini, JHEP 08, 158 (2015), 1505.07480 